\title{
Spirometry Reference Equations for Indian Children: Create Local or Go Global?
}

\author{
${ }^{*}$ LOKESh GUGLANi AND ${ }^{\#}$ DANIEL J WEINER \\ From the *Division of Pulmonology, Allergy/Immunology, Cystic Fibrosis and Sleep (PACS), Department of Pediatrics, \\ Emory University School of Medicine and Children's Healthcare of Atlanta, Atlanta GA; and ${ }^{\#}$ Division of Allergy, \\ Immunology and Pulmonology, Department of Pediatrics, Children's Hospital of Pittsburgh of UPMC, Pittsburgh PA. \\ *lokesh.guglani@gmail.com
}

S pirometry is a valuable tool for assessing patterns of lung disease, monitoring changes over time, and measuring response to interventions. Interpretation of spirometry data requires the use of reference values based on the subject's age, gender, height and ethnicity. Until recently, predicted lung function equations were plagued by their inability to account for the age-related increase in lung function in early childhood and adolescence, and for the decline in lung function with advancing age in the geriatric population. This led to the widespread use of separate sets of lung function normative data for each age-group. However, each of these datasets was created with differing inclusion criteria, equipment, measurement conditions, and acceptability standards. These differences become especially apparent as subjects move from one age category to the next and there are significant shifts in predicted values noted in changing from one dataset to another. Body composition varies between different ethnicities [1] and environmental factors have a direct correlation with lung function [2]. Hence, having a set of reference values for spirometry that have been derived from the population being tested makes the most sense. However, with increased global migration, increasing number of children with mixed race, and research collaborations between institutions, it can be helpful to avoid all the variability from different predicted values for spirometry.

The Global Lung Function Initiative (GLI) started by Quanjer [3] and Stanojevic [4] has sought to overcome the above limitations and provided a universal set of reference values for spirometry that cover ages 3 to 95 years. They are based on spirometry results from 97579 healthy, lifelong non-smokers from 72 centers in 33 countries and included the following ethnicities: Caucasians ( $n=57$ 395), African Americans $(n=3545)$, North Asian ( $n=4$ 992) and South-East Asian ( $n=8255)$. These equations were based on the LMS (lambda, mu, sigma) method, which is an extension of non-linear regression analysis that has also been used for creating growth charts. This allows the GLI equations to model the developmental changes that happen during adolescence and also provides appropriate age-dependent lower limits of normal (LLN). GLI also allows for results to be expressed as percent predicted, $Z$-scores or percentiles. A number of studies done in various populations $[5,6]$ and ethnicities around the world $[2,7,8]$ have validated the use of GLI equations across various age-groups. However, the GLI dataset does not have adequate representation from Africa, Latin America and the Indian subcontinent [9].

The manuscript by Chhabra, et al. [10] in this issue highlights the importance of adapting normative data reference equations to the adolescent growth spurt in Indian children and its direct impact on lung function. Recognizing that increase in lung function during adolescence is non-linear due to accelerated growth, they have used linear and non-linear models in their multiple regression analysis. They showed that non-linear regression matched better with the observed lung function values and were able to derive the lung function equations using multivariate analysis. They were unable to create an equation for FEV1/FVC ratio as there was no relationship with any of the independent variables. This may be a significant limitation, since the assessment of FEV1/FVC ratio is important for defining an obstructive pattern on spirometry. Having normative data for Indian children is especially important in the wake of increasing recognition of effects of ambient air pollution in urban areas of India on the growing lungs of adolescents [11.12]. Limitations of this study include being limited to a single school from where all the subjects were recruited, and not providing LLN values. In comparison, the GLI dataset includes 8255 subjects of South East Asian origin, but did not include enough subjects from the Indian subcontinent. It may be interesting to compare the 
GLI equations to those proposed by Chhabra, et al. [10], and see if any differences can be noted during adolescence.

Finally, it raises the question of which spirometry reference values should a pulmonary function testing (PFT) facility use? Most spirometry equipment comes with reference equations selected by the manufacturer and it is important for the end-user to select appropriate reference sets and to provide that information on each spirometry result. Among the 300 published reference sets that are available, only the GLI dataset provides the expanded age span coverage, the wider spectrum of ethnicities and the adjustment for adolescent growth spurt and other age-related changes. While it would be ideal for every PFT lab to generate their own normative data, it is generally not feasible and would make comparisons between different labs more difficult. Hence, it would be important to conduct additional studies to generate normative data using GLI methodology and to understand the accuracy of GLI equations for predicting the lung function of Indian children compared to the conventionally used equations.

Funding: None; Competing interest: None stated.

\section{REFERENCES}

1. Wang X, Dockery DW, Wypij D, Fay ME, Ferris BG, Jr. Pulmonary function between 6 and 18 years of age. Pediatr Pulmonol. 1993;15:75-88.

2. Sonnappa S, Lum S, Kirkby J, Bonner R, Wade A, Subramanya $\mathrm{V}$, et al. Disparities in pulmonary function in healthy children across the Indian urban-rural continuum. Am J Respir Crit Care Med. 2015;191:79-86.

3. Quanjer PH, Stanojevic S, Cole TJ, Baur X, Hall GL,
Culver BH, et al. Multi-ethnic reference values for spirometry for the 3-95-yr age range: the global lung function 2012 equations. Eur Respir J. 2012;40:1324-43.

4. Stanojevic S, Wade A, Stocks J, Hankinson J, Coates AL, Pan $\mathrm{H}$, et al. Reference ranges for spirometry across all ages: a new approach. Am J Respir Crit Care Med. 2008; 177:253-60.

5. Huls A, Kramer U, Stolz S, Hennig F, Hoffmann B, Ickstadt K, et al. Applicability of the Global Lung Initiative 2012 reference values for spirometry for longitudinal data of elderly women. PLoS One. 2016;11:e0157569.

6. Peradzynska J, Krenke K, Szylling A, Krenke R, Kulus M. The influence of the reference values on the interpretation of lung function in children: comparison of Global Lung Initiative 2012 and Polish 1998 reference values. Adv Exp Med Biol. 2015;858:31-8.

7. Pereira CA, Duarte AA, Gimenez A, Soares MR. Comparison between reference values for FVC, FEV1, and FEV1/FVC ratio in white adults in Brazil and those suggested by the Global Lung Function Initiative 2012. J Bras Pneumol. 2014;40:397-402.

8. Hall GL, Thompson BR, Stanojevic S, Abramson MJ, Beasley R, Coates A, et al. The Global Lung Initiative 2012 reference values reflect contemporary Australasian spirometry. Respirology. 2012;17:1150-1.

9. Stanojevic S, Quanjer P, Miller MR, Stocks J. The Global Lung Function Initiative: dispelling some myths of lung function test interpretation. Breathe. 2013;9:462-74.

10. Chhabra SK, Kumar R, Mittal V. Prediction equations for spirometry for children from North India. Indian Pediatr. 2016;53:781-5.

11. Kumar R, Nagar JK, Kumar H, Kushwah AS, Meena M, Kumar $\mathrm{P}$, et al. Indoor air pollution and respiratory function of children in Ashok Vihar, Delhi: an exposureresponse study. Asia Pac J Public Health. 2008;20:36-48.

12. Chattopadhyay BP, Roychowdhury A, Alam J, Kundu S. Respiratory health status of the roadside school children at Kolkata. J Environ Sci Eng. 2005;47:202-11. 